\title{
Cárie dentária e fatores associados em crianças de 5 anos de idade do município de Patos-PB
}

\author{
Dental caries and associated factors in children 5-year-old children \\ from the county Patos- $P B$ \\ Caries dental y factores asociados en niños de 5 años de edad \\ del municipio Patos-PB \\ Faldryene de Sousa QUEIROZ ${ }^{1}$ \\ Luciana Ellen Dantas COSTA ${ }^{2}$ \\ Kaline Lays Silva SANTOS ${ }^{2}$ \\ Thamyres Maria Silva SIMÕES ${ }^{2}$ \\ Paula Vanessa da SILVA ${ }^{3}$ \\ ${ }^{1}$ Professora Doutora do Curso de Odontologia, Unidade Acadêmica de Ciências Biológicas, Centro de Saúde e Tecnologia Rural, \\ Área de Saúde Coletiva, Universidade Federal de Campina Grande -UFCG 58708-110, Patos - PB, Brasil \\ ${ }^{2}$ Unidade Acadêmica de Ciências Biológicas, Centro de Saúde e Tecnologia Rural, Universidade Federal de Campina Grande -UFCG \\ 58708-110, Patos - PB, Brasil \\ ${ }^{3}$ Departamento de Odontologia, Faculdades Integradas de Patos - FIP 58700-000, Patos - PB, Brasil
}

\section{Resumo}

O presente estudo teve como objetivo analisar a associação entre cárie dentária, hábitos alimentares e de higiene e a percepção dos pais sobre saúde bucal de crianças na faixa etária de 5 anos de idade do município de Patos-PB. A pesquisa foi realizada com 243 crianças de 5 anos de idade, matriculadas em creches públicas do município de Patos/PB e seus respectivos pais/responsáveis. Para a coleta dos dados utilizou-se os índices IHOS e ceo-d, e aplicou-se questionários aos pais avaliando hábitos alimentares e de higiene e sua percepção sobre saúde bucal. Para avaliar a associação entre a cárie dentária e os fatores associados utilizou-se o teste do QuiQuadrado $(\mathrm{p}<0,05)$. O índice ceo-d médio foi de 3,11. Houve a associação significativa entre a cárie e o índice de higiene oral $(\mathrm{p}=0,016)$, uso da mamadeira $(\mathrm{p}=0,016)$ e a percepção dos pais se a cárie é transmissível $(\mathrm{p}=0,003)$. É alta a prevalência de cárie em pré-escolares do município de Patos-PB e os hábitos alimentares, de higiene bucal, bem como o conhecimento dos cuidadores sobre o tema, podem influenciar diretamente no aparecimento da doença.

Descritores: Cárie Dentária; Pré-Escolar; Saúde Bucal.

\begin{abstract}
The present study aimed to analyze the association between dental caries, food and hygiene habits and the perception of parents about oral health of children aged 5 years old in the city of Patos-PB. The study was conducted with 243 children under 5 years of age, enrolled in public kindergartens in the city of Patos/PB and their parents/guardians. To collect the data we used the IHOS and ceo-d, and applied questionnaires to parents about the eating habits and health and your oral health perception. To evaluate the association between dental caries and associated factors, the Chi-Square test was used $(\mathrm{p}<0.05)$. The ceo-d average rate was 3,11 . There was a significant correlation between dental caries and oral hygiene index $(p=0.016)$, bottle-feeding $(p=0.016)$ and the perception of parents if the decay is transmitted $(\mathrm{p}=0.003)$. Finally, it is concluded that it is still a high prevalence of dental caries in preschool children and eating habits, oral hygiene, as well as knowledge of caregivers on the topic, can directly influence the onset of the disease.

Descriptors: Dental Caries; Child, Preschool; Oral Health.
\end{abstract}

\section{Resumen}

El presente estudio tuvo como objetivo analizar la asociación entre caries, hábitos alimenticios y de higiene y la percepción de los padres sobre salud bucal de niños en el grupo de edad de 5 años de edad del municipio de Patos-PB. La encuesta fue realizada con 243 niños de 5 años de edad, matriculados en guarderías públicas del municipio de Patos / PB y sus respectivos padres / responsables. Para la recolección de los datos se utilizaron los índices IHOS y ceo-d, y se aplicó cuestionarios a los padres evaluando hábitos alimentarios y de higiene y su percepción sobre salud bucal. Para evaluar la asociación entre la caries dental y los factores asociados se utilizó la prueba del Qui-Cuadrado $(\mathrm{p}<0,05)$. El índice ceo-d medio fue de 3,11. Se observó una asociación significativa entre la caries y el índice de higiene oral $(\mathrm{p}=0,016)$, el uso del biberón $(\mathrm{p}=0,016)$ y la percepción de los padres si la caries es transmisible $(\mathrm{p}=0,003)$. Es alta la prevalencia de caries en preescolares del municipio de Patos-PB y los hábitos alimentarios, de higiene bucal, así como el conocimiento de los cuidadores sobre el tema, pueden influenciar directamente en la aparición de la enfermedad.

Descriptores: Caries Dental; Preescolar; Salud Bucal.

\section{INTRODUÇÃO}

A cárie dentária é uma patologia de caráter multifatorial, que ocorre através da interação entre a dieta, hospedeiro (dente) e bactérias, somados ao fator tempo para que ocorra a doença ${ }^{1}$. Revela-se um grande desafio para a saúde pública em nível mundial, por ser a doença crônica mais comum na infância ${ }^{2}$.

Nos dois últimos levantamentos epidemiológicos, feitos em 2002/2003 e 2010 pelo SB BRASIL, pôde-se observar um aumento de cerca de $6 \%$ de crianças de cinco anos de idade livres de cárie ${ }^{3}$. Apesar desta pequena melhoria, as condições de saúde bucal de crianças em idade pré-escolar, no Brasil, ainda são preocupantes ${ }^{4}$.

Múltiplos fatores podem ser apontados como possíveis causas para o declínio da doença: a fluoretação das águas de abastecimento público, o emprego de dentifrícios fluoretados em larga escala, modificações no padrão e quantidade de consumo de açúcar, associados à melhoria nas condições de vida, e maior acesso à atenção em saúde bucal coletiva, a partir da ampliação das ações de promoção e educação em saúde bucal ${ }^{5}$.

A doença cárie afeta desproporcionalmente a população economicamente desfavorecida. A inacessibilidade a cuidados dentários, baixos níveis de flúor da água da comunidade e a falta de conhecimento dos pais sobre saúde bucal são fatores que influenciam diretamente em uma dieta e higiene oral inadequada, favorecendo assim, o aparecimento da cárie dentária ${ }^{1}$.

É na infância onde se inicia o desenvolvimento de hábitos alimentares e estes influenciam diretamente nas escolhas e no modo de se alimentar do indivíduo ao longo de todo o ciclo de vida. No início do período de diversificação alimentar é onde as mães tem seu papel fundamental e são as principais responsáveis pela introdução de alimentos saudáveis ou não na dieta da criança ${ }^{6}$.

Desta forma, acredita-se na importância de conhecer as problemáticas envolvidas na manutenção da saúde bucal da 
criança em fase pré-escolar, no âmbito biológico e social ao qual está inserida, pois é nesta fase em que os cuidados primários promovem a prevenção de futuras cáries dentárias.

Portanto, o presente estudo teve como objetivo analisar a associação entre cárie dentária, hábitos alimentares e de higiene e a percepção dos pais sobre saúde bucal de crianças na faixa etária de 5 anos de idade do município de Patos-PB.

\section{MATERIAL E MÉTODO}

O estudo caracterizado como observacional e quantitativo, através de levantamento de dados, foi conduzido dentro dos padrões exigidos pela declaração de Helsink e aprovado pelo Comitê de Ética em Pesquisa com Seres Humanos da Universidade Cruzeiro do Sul (Parecer $n^{\circ}$ 027/2014).

Participaram do estudo 243 crianças de 5 anos matriculadas em creches públicas do município de Patos/PB e seus respectivos pais/responsáveis, após assinatura do Termo de Consentimento Livre e Esclarecido (TCLE).

O município de Patos/PB conta com 12 creches públicas divididas em cinco regiões de ensino e um distrito. Para obtenção da amostra, realizou-se a seleção das creches por amostragem probabilística casual simples sem reposição, sendo sorteada uma creche por região de ensino por distrito, totalizando 6 creches municipais às quais foram utilizadas para obtenção dos pré-escolares.

O tamanho da amostra do estudo foi obtido por meio do processo de amostragem para população finita ${ }^{7}$. A margem de erro fixada e valor admitido para média $(x=3,11)$ e desviopadrão $(\delta=3,26)$ foram os mesmos adotados pelo Projeto Saúde Bucal Brasil 2010 (Projeto SBBrasil) para a região Nordeste, para crianças de 5 anos de idade, em população maior de 100 mil habitantes ${ }^{3}$. O nível de significância adotado foi de 5\%. Nestas condições o tamanho amostral ficou estimado em 212 escolares. Admitindo-se um absenteísmo da ordem de $20 \%$, o tamanho final da amostra foi de 254 préescolares.

Foram excluídas da amostra crianças que apresentaram deficiências neuropsicomotoras, que não permitiram a realização do exame clínico ou que foram vetadas pelos pais/responsáveis e aquelas em que não houve o preenchimento do termo de consentimento livre e esclarecido e do questionário.

Previamente à execução da pesquisa foram realizadas visitas às creches ao qual foram apresentadas, aos diretores das escolas e aos pais ou responsáveis das crianças, as proposições do estudo, assim como foi solicitada a autorização dos mesmos para a condução da pesquisa por meio da distribuição dos Termos de Consentimento Livre e Esclarecido.

Para coleta de dados, os questionários foram entregues aos pais/responsáveis para responderem sobre as condições socioeconômicas, hábitos alimentares e de higiene e a percepção dos pais sobre saúde bucal.

Os exames clínicos foram realizados por um examinador e anotador previamente calibrados. Os préescolares foram examinados após escovação supervisionada e utilizados gazes para secagem das superfícies dentárias, espelhos bucais e sonda periodontal da OMS (sonda CPI) para levantamentos epidemiológicos, nas dependências das creches em local sob iluminação natural, ventilado e próximo de uma fonte de água, estando examinador e criança sentados em cadeiras ${ }^{8}$.

Para avaliação das condições de saúde bucal dos préescolares foi utilizado o índice ceo-d, segundo os códigos e critérios recomendados pela Organização Mundial da Saúde ${ }^{8}$. Para análise dos níveis de placa, os dados foram obtidos através do Índice de Higiene Oral Simplificado (IHOS) criado por Greene e Vermillion ${ }^{9}$ em 1964.
A análise estatística foi realizada de modo descritivo por meio de frequências relativas e absolutas para as variáveis categóricas e o teste do Qui-quadrado foi utilizado para verificar as associações entre a presença cárie com os fatores associados. Para a realização dos testes estatísticos foi adotado nível de significância de 5\%. Os testes foram calculados por meio do programa SPSS ${ }^{\circledR}$ (Statistical Package for the Social Sciences) versão 21.0 para Microsoft Windows.

\section{RESULTADOS}

\section{- Caracterização Amostral}

$O$ total de crianças e seus respectivos pais/responsáveis participantes desse estudo foi de 243, correspondendo a $95,6 \%$ do total selecionado mediante a realização do cálculo amostral. A perda de 11 crianças ocorreu devido a: ausência da criança na creche no dia de realização do exame clínico e/ou falta de colaboração por parte das crianças na realização do exame.

Das 243 crianças participantes, a maioria de 54,3\% $(\mathrm{n}=132)$ foi do sexo masculino. $\mathrm{O}$ índice ceo-d variou de $0 \mathrm{a}$ 14, com média de 3,11 considerada de prevalência moderada.

Ao analisarmos o perfil familiar dos pais/responsáveis entrevistados, vimos que $79,4 \%(n=193)$ afirmaram receber assistência social do Governo Federal, e destas, 77\% ( $\mathrm{n}=187)$ recebem o auxílio do Bolsa Família. Em relação à escolaridade, $91,4 \% \quad(n=222)$ são alfabetizados, no qual 48,5\% ( $n=118)$ apresentam apenas o Ensino Fundamental Completo. Infelizmente, mesmo sendo a minoria, mas ainda significante, $8,6 \%(\mathrm{n}=21)$ dos responsáveis são analfabetos. Também verificou-se a quantidade de pessoas que moram na mesma casa e $77,8 \%(n=189)$ relataram haver menos de 6 pessoas convivendo no mesmo ambiente familiar.

○ Análise Bivariada

Foi avaliada a presença de cárie em função dos hábitos alimentares e de higiene bucal, apresentada na Tabela 1, e a prevalência de cárie em função da percepção dos pais/responsáveis sobre saúde bucal, apresentada na Tabela 2 , pelo teste Qui-Quadrado $(\mathrm{p}<0,05)$.

$\mathrm{Na}$ Tabela 1 , em relação às variáveis de hábitos de higiene bucal, observou-se uma relação estatisticamente significante $(\mathrm{p}=0,016)$ entre a presença de cárie dentária em função do índice de higiene oral, no qual 77,6\% (n=38) das crianças com presença de cárie apresentavam IHOS deficiente.

Embora a relação entre cárie dentária e a frequência de escovação por dia não tenha sido estatisticamente significante $(\mathrm{p}=0,522)$, observou-se que $67,7 \%(\mathrm{n}=90)$ das crianças com presença de cárie escovavam os dentes até no máximo duas vezes ao dia.

$\mathrm{Na}$ análise relacionada aos hábitos alimentares, apenas a variável do uso da mamadeira foi associada à presença de cárie, no qual se mostrou estatisticamente significante $(p=0,016)$, visto que $63,9 \%(n=115)$ das crianças que fizeram ou fazem o uso da mamadeira apresentavam cárie dentária.

Alguns resultados também se mostraram relevantes ao estudo, porém não estatisticamente significantes, como no caso das variáveis relacionadas à idade em que a criança começou a consumir alimentos açucarados $(p=0,119)$, a duração do aleitamento materno $(p=0,474)$ e a duração do uso da mamadeira $(p=0,383)$ em detrimento da presença ou ausência de cárie. Realizado o cruzamento dos dados, observou-se que $65 \%(n=102)$ das crianças que começaram a consumir açúcar na alimentação com menos de 1 ano de idade apresentavam cárie dentária. A doença cárie também se revelou presente em 73,6\% (n=39) das crianças que mamaram por mais de 2 anos de idade e em 69\% (n=98) das crianças que fizeram o uso da mamadeira por este mesmo período de tempo. 
Tabela 1. Presença de cárie em função dos hábitos alimentares e de higiene bucal, Patos-PB, 2017

\begin{tabular}{|c|c|c|c|c|}
\hline \multirow[b]{2}{*}{ Variável } & \multicolumn{3}{|c|}{ CÁRIE DENTÁRIA } & \multirow[b]{2}{*}{ Valor de $\mathbf{p}^{(1)}$} \\
\hline & $\begin{array}{c}\text { Ausente } \\
\mathrm{n}(\%)\end{array}$ & $\begin{array}{c}\text { Presente } \\
\mathrm{n}(\%)\end{array}$ & $\begin{array}{l}\text { Total } \\
\mathrm{n}(\%)\end{array}$ & \\
\hline \multicolumn{5}{|c|}{ Hábitos de higiene bucal } \\
\hline \multicolumn{5}{|l|}{ IHOS } \\
\hline $\begin{array}{l}\text { Bom } \\
\text { Regular } \\
\text { Deficiente }\end{array}$ & $\begin{array}{l}10(55,6) \\
57(32,4) \\
11(22,4)\end{array}$ & $\begin{array}{r}8(44,4) \\
119(67,6) \\
38(77,6)\end{array}$ & $\begin{array}{c}18(100,0) \\
176(100,0) \\
49(100,0)\end{array}$ & $0,016^{*}$ \\
\hline \multicolumn{5}{|c|}{ É utilizado creme dental durante a escovação? } \\
\hline $\begin{array}{l}\text { Sim } \\
\text { Não }\end{array}$ & $\begin{array}{l}76(31,7) \\
2(66,7)\end{array}$ & $\begin{array}{c}164(68,3) \\
1(33,3)\end{array}$ & $\begin{array}{c}240(100,0) \\
3(100,0)\end{array}$ & 0,242 \\
\hline $\begin{array}{l}\text { Em relação à quantida } \\
\text { Cobre totalmente as } \\
\text { cerdas da escova } \\
\text { Cobre metade das } \\
\text { cerdas da escova } \\
\text { Cobre menos da } \\
\text { metade das cerdas da } \\
\text { escova }\end{array}$ & $\begin{array}{l}\text { Ide de crem } \\
33(34,0)\end{array}$ & $53(66,2)$ & $\begin{array}{l}80(100,0) \\
66(100,0)\end{array}$ & 0,392 \\
\hline $\begin{array}{l}\text { Como seu filho realiza } \\
\text { Com supervisão de um } \\
\text { adulto } \\
\text { Sem supervisão de um } \\
\text { adulto }\end{array}$ & $\begin{array}{l}\text { a escovaç } \\
50(31,1) \\
28(34,1)\end{array}$ & $\begin{array}{l}111(68,9) \\
54(65,9)\end{array}$ & $\begin{array}{l}161(100,0) \\
82(100,0)\end{array}$ & 0,364 \\
\hline $\begin{array}{l}\text { Com qual frequência a } \\
\leq \text { a } 2 \text { vezes por dia } \\
\geq \text { a } 3 \text { vezes por dia }\end{array}$ & $\begin{array}{l}\text { criança es } \\
43(32,3) \\
35(31,8)\end{array}$ & $\begin{array}{c}\text { cova os de } \\
90(67,7) \\
75(68,2)\end{array}$ & $\begin{array}{c}\text { tes por dia? } \\
133(100,0) \\
110(100,0)\end{array}$ & 0,522 \\
\hline \multicolumn{5}{|l|}{ Hábitos alimentares } \\
\hline $\begin{array}{l}\text { Com que idade seu fill } \\
<1 \text { ano de idade } \\
\geq 1 \text { ano de idade }\end{array}$ & $\begin{array}{l}\text { ho começo } \\
55(35,0) \\
23(26,7)\end{array}$ & $\begin{array}{c}\text { u a consum } \\
102(65,0) \\
63(73,3)\end{array}$ & $\begin{array}{c}\text { ir alimentos a } \\
157(100,0) \\
86(100,0)\end{array}$ & $\begin{array}{c}\text { çucarados? } \\
0,119\end{array}$ \\
\hline $\begin{array}{l}\text { Quantas vezes por dia } \\
\leq \text { a } 2 \text { vezes ao dia } \\
\text { za } 3 \text { vezes ao dia } \\
\text { Não informado }\end{array}$ & $\begin{array}{l}\text { a criança } \\
49(30,2) \\
27(38,6) \\
2(18,2)\end{array}$ & $\begin{array}{c}\text { consome al } \\
113(69,8) \\
43(61,4) \\
9(81,8)\end{array}$ & $\begin{array}{c}\text { mentos açuc } \\
162(100,0) \\
70(100,0) \\
11(100,00)\end{array}$ & $\begin{array}{l}\text { arados? } \\
\qquad 0,267\end{array}$ \\
\hline \multicolumn{5}{|l|}{$\begin{array}{l}\text { Seu filho foi } \\
\text { amamentado? }\end{array}$} \\
\hline $\begin{array}{l}\text { Duração do aleitament } \\
\leq 1 \text { ano de idade } \\
>2 \text { anos de idade } \\
\text { Não informado }\end{array}$ & $\begin{array}{l}\text { to materno: } \\
52(34,9) \\
14(26,4) \\
12(29,3)\end{array}$ & $\begin{array}{l}97(65,1) \\
39(73,6) \\
29(70,7)\end{array}$ & $\begin{array}{l}149(100,0) \\
53(100,0) \\
41(100,0)\end{array}$ & 0,474 \\
\hline $\begin{array}{l}\text { Seu filho usou } \\
\text { mamadeira? } \\
\text { Sim } \\
\text { Não }\end{array}$ & \multicolumn{3}{|c|}{$\begin{array}{l}\text { Seu filho usou } \\
\text { mamadeira? }\end{array}$} & $0,016^{*}$ \\
\hline $\begin{array}{l}\text { Duração do uso da ma } \\
\leq 1 \text { ano de idade } \\
>2 \text { anos de idade } \\
\text { Não informado }\end{array}$ & $\begin{array}{l}\text { madeira: } \\
14(42,4) \\
44(31,0) \\
20(29,4)\end{array}$ & $\begin{array}{l}19(57,6) \\
98(69,0) \\
48(70,6)\end{array}$ & $\begin{array}{c}33(100,0) \\
142(100,0) \\
68(100,0)\end{array}$ & 0,383 \\
\hline
\end{tabular}

$\mathrm{Na}$ Tabela 2 houve associação estatisticamente significante $(\mathrm{p}=0,003)$ entre a presença de cárie e a percepção do pai/responsável sobre a transmissibilidade da cárie, uma vez que $58,3 \% \quad(n=63)$ dos pais que não tinham conhecimento de que a cárie poderia passar de pessoa para pessoa, tiveram seus filhos acometidos pela doença cárie.

Ao questionarmos aos pais/responsáveis se o consumo de açúcar em excesso poderia ser prejudicial à saúde bucal, apesar de não haver associação estatisticamente significante $(p=0,285)$, observamos que $72,7 \%(n=32)$ responderam que não e os filhos destes respectivos pais/responsáveis apresentavam cárie dentária.

Acerca da variável à qual se pergunta aos pais se já receberam alguma informação sobre higiene bucal em relação à presença ou ausência de cárie em seus filhos, observou-se que $69,9 \%(n=51)$ dos pais que não tiveram acesso a qualquer informação também revelaram presença de cárie na cavidade oral das crianças, embora essa relação não tenha sido estatisticamente significante $(\mathrm{p}=0,393)$.
Tabela 2. Presença de cárie em função da percepção dos pais/responsáveis sobre saúde bucal, Patos-PB, 2017

\begin{tabular}{|c|c|c|c|c|}
\hline \multirow[b]{2}{*}{ Variável } & \multicolumn{3}{|c|}{ CÁRIE DENTÁRIA } & \multirow[b]{2}{*}{ Valor de $\mathbf{p}^{(1)}$} \\
\hline & $\begin{array}{c}\text { Ausente } \\
\mathrm{n}(\%)\end{array}$ & $\begin{array}{c}\text { Presente } \\
\mathrm{n}(\%)\end{array}$ & $\begin{array}{l}\text { Total } \\
\mathrm{n}(\%)\end{array}$ & \\
\hline \multicolumn{5}{|c|}{ O consumo de açúcar em excesso pode trazer prejuízos à saúde bucal? } \\
\hline $\begin{array}{l}\text { Sim } \\
\text { Não }\end{array}$ & $\begin{array}{l}66(33,2) \\
12(27,3)\end{array}$ & $\begin{array}{c}133(66,8) \\
32(72,7)\end{array}$ & $\begin{array}{c}199(100,0) \\
44(100,0)\end{array}$ & 0,285 \\
\hline \multicolumn{5}{|c|}{ Você considera a cárie como doença? } \\
\hline Sim & $63(30,1)$ & $146(69,9)$ & $209(100,0)$ & 0,080 \\
\hline Não & $15(44,1)$ & $19(55,9)$ & $34(100,0)$ & \\
\hline \multicolumn{5}{|c|}{ Você acha que a cárie pode passar de pessoa para pessoa? } \\
\hline $\operatorname{Sim}$ & $33(24,4)$ & $102(75,6)$ & $135(100,0)$ & $0,003^{\star}$ \\
\hline Não & $45(41,7)$ & $63(58,3)$ & $108(100,0)$ & \\
\hline \multicolumn{5}{|c|}{ Saúde bucal pode afetar a saúde geral? } \\
\hline Sim & $69(32,1)$ & $146(67,9)$ & $215(100,0)$ & 0,575 \\
\hline Não & $9(32,1)$ & $19(67,9)$ & $28(100,0)$ & \\
\hline \multicolumn{5}{|c|}{ Você já recebeu alguma informação sobre higiene bucal? } \\
\hline Sim & $56(32,9)$ & $114(67,1)$ & $170(100,0)$ & 0,393 \\
\hline Não & $22(30,1)$ & $51(69,9)$ & $73(100,0)$ & \\
\hline \multicolumn{5}{|c|}{ Algum membro da família foi ao dentista nos últimos 12 meses? } \\
\hline Sim & $54(31,4)$ & $118(68,6)$ & $172(100,0)$ & 0,617 \\
\hline Não & $24(34,3)$ & $47(65,7)$ & $71 \quad 100,0$ & \\
\hline
\end{tabular}

(1) Teste Qui-quadrado/*Significante ao nível de $5 \%(\mathrm{p}<0,05)$

\section{DISCUSSÃO}

No presente estudo, demonstrou-se que as crianças apresentaram prevalência moderada de cárie dentária, com ceo-d médio de 3,11. De acordo com o SB Brasil 2010², as médias encontradas em crianças com 5 anos de idade em âmbito nacional e na cidade de João Pessoa, capital da Paraíba, foi de 2,43 e 2,61, respectivamente, dados aquém aos encontrados neste estudo. E na região nordeste o ceo-d médio encontrado é de $2,89^{10}$. Porém, o valor do ceo-d nas cidades do interior do nordeste foi de 3,94, corroborando com os dados obtidos na pesquisa ${ }^{3}$.

A presente pesquisa apresentou uma correlação estatisticamente significante entre o IHOS e a cárie dentária. A relação entre a higienização não adequada, caracterizada pela presença de placa visível, e o desenvolvimento da cárie em crianças já é bem esperada ${ }^{11}$. Corroborando com estes dados, observou-se neste estudo que a maioria das crianças que escovavam os dentes menos de duas vezes ao dia apresentaram maior percentual de presença de cárie dentária. $\mathrm{Na}$ mesma linha de resultado, outros estudos também identificaram correlação entre maior prevalência de cárie associada à presença de placa visível e à baixa frequência de escovação dental infanti1 ${ }^{11,12}$. Desta forma, pode-se afirmar que a falta de escovação tem influência sobre a manutenção de uma boa higiene oral e ao desenvolvimento de cárie dentária.

A introdução precoce de açúcar e de alimentos que contenham açúcar na fase da formação dos hábitos da criança pode levar ao maior consumo destes alimentos no decorrer da vida, influenciando diretamente na saúde bucal da criança e facilitando o desenvolvimento da doença cárie ${ }^{13}$. No presente estudo observou-se que a cárie se mostrou presente nas crianças que começaram a consumir açúcar precocemente, antes de 1 ano de idade. Afirmação também comprovada nos estudos propostos por Moura et. al. ${ }^{13}$, Cangussu et. al. ${ }^{14} \mathrm{e}$ Coclete et. al. ${ }^{15}$.

No entanto, não foi encontrada correlação da cárie com o estado nutricional no estudo feito por Porcelli et. al. ${ }^{16}$, pois o caráter multifatorial da doença cárie precisava ser elucidado, como a quantidade e qualidade da escovação, condição socioeconômica da família e baixa escolaridade da mãe que influenciam a escolha de hábitos alimentares inadequados. Contudo, estes fatores foram explanados neste estudo, pois a maioria dos pais/responsáveis recebe auxílio do Governo Federal e em relação ao grau de escolaridade da 
mãe, o ensino fundamental completo foi a resposta mais encontrada, reforçando o resultado inicial encontrado.

$\mathrm{O}$ aleitamento materno, tanto quanto o desmame, tem sua importância para o bom desenvolvimento da criança, porém, devemos ficar atentos, pois a presença de cárie dentária se encontra diretamente associada ao aleitamento materno quando o padrão de consumo apresenta determinadas características, como livre demanda, frequência elevada de mamadas ao dia, longa duração das mamadas e, principalmente, mamadas noturnas frequentes, levando ao acúmulo de leite sobre os dentes, associado à redução de fluxo salivar e à ausência de limpeza dos dentes ${ }^{17}$.

Afirmação comprovada neste trabalho, uma vez que se pôde constatar uma correlação entre presença de cárie e uso da mamadeira, sendo também ressaltada pelos resultados observados na associação entre as durações do uso da mamadeira e do aleitamento materno por mais de 2 anos de idade com o desenvolvimento da cárie. Na mesma linha de resultado, diversos estudos verificaram associação entre a amamentação por períodos mais longos que 12 meses e um aumento de 2 a 3 vezes na ocorrência de cárie dentária em dentes decíduos ${ }^{14,15,18}$.

No que diz respeito à percepção dos pais/responsáveis sobre saúde bucal, a maioria destes informaram que a cárie não passa de pessoa para pessoa. A falta de conhecimento leva à falta de cuidados com a saúde bucal dos filhos, o que pode se afirmar com a presença de cárie encontrada na maioria das crianças desta pesquisa, e em um estudo semelhante, no interior da Bahia, no qual foi realizado o mesmo questionamento e houve a associação em $60,9 \%$ das famílias pesquisadas ${ }^{19}$. Porém, deve-se salientar que o que é transmissível é o microorganismo cariogênico ( $S$. mutans), apontado como principal agente etiológico da cárie dental em humanos, devendo os pais evitarem beijar a boca dos filhos, assoprar o alimento antes de levar à criança, pois, desta forma, a bactéria pode sim ser transmitida.

Ao questionar os pais/responsáveis acerca do consumo de açúcar em excesso ser prejudicial à saúde bucal, observouse que houve associação entre aqueles que responderam que não há relação de prejuízo com a presença de cárie encontrada em seus filhos. A busca por conhecimento sobre saúde bucal por parte dos cuidadores é fundamental para a melhora dos padrões de saúde bucal nas crianças. No presente estudo a maioria dos pais afirmaram nunca ter obtido qualquer orientação sobre saúde bucal, o que remete a necessidade de intensificar os programas educativos/ preventivos na área de saúde pública.

\section{CONCLUSÃO}

É alta a prevalência de cárie em pré-escolares do município de Patos-PB e os hábitos alimentares, de higiene bucal, bem como o conhecimento dos cuidadores sobre o tema, podem influenciar diretamente no aparecimento da doença. Faz-se necessário um maior incentivo a atividades de promoção e prevenção em saúde bucal no intuito de esclarecer ainda mais a população sobre os fatores relacionados ao desenvolvimento da cárie dentária.

\section{REFERÊNCIAS}

1. Chou R, Cantor A, Zakher B, Mitchell JP, Pappas M. Preventing dental caries in children $<5$ years: systematic review up dating USPSTF recommendation. Pediatrics. 2013; 132(2):332-50.

2. Lopes TR, Almeida ABD, Moreira RDO, Carvalho AAH, Garcia FDM, Rocha CM et al. Determinantes sociais e biológicos da cárie dentária na infância: Uma experiência interdisciplinar no PET Saúde-UFJF. Rev APS. 2015; 18(1):30-8.

3. Brasil. Ministério da Saúde. Secretaria de Atenção à Saúde. Secretaria de Vigilância em Saúde. SB Brasil
2010: Pesquisa Nacional de Saúde Bucal: resultados principais / Ministério da Saúde , 2012; Secretaria de Atenção à Saúde. Secretaria de Vigilância em Saúde. Brasília: Ministério da Saúde.

4. Souza JGS, Martins AMEBL. Dor dentária e fatores associados em pré-escolares brasileiros. Rev Paul Pediatr. 2016; 34(3):336-42.

5. Barbosa APM, Kriger L, Moysés ST, Moysés SJ. Prevalência da doença cárie em crianças de cinco anos de idade na cidade de Curitiba-análise crítica. Epidemiol Serv Saúde. 2007; 16(2):142-5.

6. Scaglioni S, Salvioni M, Galimberti C. Influence of parental attitudes in the development of children eating behaviour. Br J Nutr. 2008; 99(Suppl 1): S22-5.

7. Cochran WG. Sampling Techniques. 3 ed. Nova Iorque: Wiley; 1977.

8. OMS. Levantamentos básicos em saúde bucal. São Paulo: Santos; 1999.

9. Greene JG, Vermillion JR. The simplified oral hygiene index. J Am Dent Assoc. 1964; 68:7-13.

10. Mendes HJ, Matos PES, Bastos JRM. Cárie dentária e desigualdades socioeconômicas no Brasil. Rev Saúde Com. 2016; 12(1):454-62 .

11. Correa-Faria P, Martins-Junior PA, Vieira-Andrade, RG, Marques LS, Ramos-Jorge ML. Factors associated with the development of early childhood caries among Brazilian preschoolers. Braz Oral Res. 2013; 27(4):356-62.

12. Gomes VE, da Silva DD. A importância do controle de placa dental na clínica odontológica. Arq Odontol. 2016; 46(1):22-7.

13. Moura SMS, Oliveira IM, Leite CMC, Conde Júnior AM. Dieta e cárie dental em escolares de 10 a 14 anos na cidade de Picos, Piauí. J Health Sci. 2016; 18(1):18-22.

14. Cangussu MC, Cabral MBBS, Mota ELA, Vianna MIP. Fatores de risco para a cárie dental em crianças na primeira infância, Salvador - BA. Rev Bras Saúde Matern Infant. 2016; 16(1):57-65.

15. Coclete GEG, Nagata ME, Hosida TY, Báez-Quintero LC, Cunha RF, Pessan JP. Cárie precoce da infância: reabilitação estética e funcional. Arch Health Invest. 2016; 5(Spec Iss 1): 237.

16. Porcelli ICS, Braga MP, Corsi NM, Poli-Frederico RC, Maciel SM. Prevalência da cárie dentária e sua relação com as condições nutricionais entre escolares de um município do sul do Brasil. ClipeOdonto. 2016; 8(1):2-9.

17. Santos AP, Nadanovsky P, Oliveira BH. A systematic review and meta-analysis of the effects of fluoride toothpastes on the prevention of dental caries in the primary dentition of preschool children. Community Dent Oral Epidemiol. 2013; 41(1):1-12.

18. Victora CG, Bahl R, Barros AJ, França GV, Horton S, Krasevec $J$ et al. Breastfeeding in the 21st century: epidemiology, mechanisms, and lifelong effect. Lancet. 2016; 387(10017):475-90.

19. Oliveira ASS, Uemura TF. Cárie dentária em crianças de um município da Bahia e conhecimento dos seus responsáveis sobre saúde bucal. Rev Saúde Com. 2016; 12(2):535-41. 


\section{CONFLITO DE INTERESSES}

Os autores declaram não haver conflitos de interesse.

\section{AUTOR PARA CORRESPONDÊNCIA}

Faldryene de Sousa Queiroz

falqueiroz@hotmail.com

Submetido em 27/02/2018

Aceito em 09/04/2018 